\title{
AN OPERATIONAL CALCULUS FOR INFINITESIMAL GENERATORS OF SEMIGROUPS
}

\author{
BY

\section{A. V. BALAKRISHNAN}

The primary feature of any operational calculus is the extension of an algebra of numerically valued functions $f(\lambda)$, an algebra of operators $f(A)$ over a $B$-space, where $A$ is a suitably restricted linear operator. In this paper the operator $A$ is taken to be the infinitesimal generator of a strongly continuous semigroup $\left({ }^{1}\right) T(\xi)$ of linear bounded transformations over a $B$-space $X$. With this restriction on $A$, which is natural for the applications we have in mind, we are able to let the operators $f(A)$ be unbounded.

If $f(\lambda)$ is a Laplace-Stieltjes transform, i.e., if

$$
f(\lambda)=\int_{0}^{\infty} e^{\lambda \xi} d \xi \alpha
$$

one might, with the heuristic correspondence principle $e^{\lambda \xi} \sim T(\xi)$ in mind, define $f(A)$ by

$$
f(A) x=\int_{0}^{\infty} T(\xi) x d_{\xi} \alpha, \quad x \in X
$$

$\lambda, \alpha$ being suitably restricted for the convergence of the integrals involved. Such a calculus was first described by Hille [1] and has been extensively developed by Phillips $[2 ; 3 ; 4]$. In the Hille-Phillips calculus the operators $f(A)$ are bounded operators, and for many purposes it would be convenient to have a calculus in which the $f(A)$ is not restricted to be bounded. This paper describes one way of extending the Hille-Phillips calculus to this end. [See the reference in [2] for an alternate point of view due to L. Schwartz.] More explicitly, we describe a calculus in which the functions $f(\lambda)$ are ratios of Laplace transforms, and the operators $f(A)$ are closed linear with dense domain.

We begin in $\$ 2$, with a summary of some of the basic facts concerning semigroups of operators, including a brief description of the Hille-Phillips calculus. The extended calculus is described in $\S 3$. In $\$ 4$ we take up spectral theory, the sine qua non of any operational calculus. $\$ 5$ deals with semigroups of convolution operators. Thus, if $\alpha(t), t>0$, is a one-parameter family of

Presented to the Society, December 28, 1954, under the title An operational calculus for closed operators; received by the editors July 29, 1957.

(1) For the relevant material on semigroup theory see Hille [1]. See also [1] for the literature on Operational Calculi. 
countably additive set functions defined on the Borel sets of the half-line $[0, \infty)$, forming a semigroup under convolution in $t$, we obtain a new semigroup over $X$, if we set, using (1.1)

$$
S(t) x=\int_{0}^{\infty} T(\xi) x d_{\xi} \alpha(t), \quad x \in X .
$$

The generation of such semigroups is of importance for instance in stochastic process theory. The relationship of the infinitesimal generator of the semigroup $S(t)$ to that of $T(\xi)$ is of primary interest here, and is a problem to which the extended calculus is particularly suited. Finally, in $\$ 6$, we include an application of the calculus and indicate how it may be used to generate nonintegral powers of the infinitesimal generator of a semigroup.

2. Preliminaries. Let $T(\xi), 0<\xi$, be a one-parameter semigroup of linear bounded operators over a $B$-space $X$, such that:

$$
\begin{aligned}
& \text { (i) } \quad T\left(\xi_{1}+\xi_{2}\right)=T\left(\xi_{1}\right) T\left(\xi_{2}\right), \quad \xi_{1}, \xi_{2}>0 \\
& T(0)=I \text { (Identity) } \\
& \text { (ii) }\|T(\xi) x-x\| \rightarrow 0 \text { as } t \rightarrow 0, \quad x \in X \\
& w^{+}=\operatorname{Inf}_{\xi>0} \frac{\log \|T(\xi)\|}{\xi}>-\infty .
\end{aligned}
$$

Then $w^{+}<\infty$, [see [1] for these and other properties that follow] and for every $\sigma>0$, we can find an $M(\sigma)<\infty$ such that

$$
\|T(\xi)\| \leqq M(\sigma) \operatorname{Exp}\left(w^{+}+\sigma\right) \xi .
$$

The infinitesimal generator $A$ is defined by:

$$
A x=\operatorname{limit}_{\xi \rightarrow 0} \frac{T(\xi) x-x}{\xi}
$$

and is a closed linear operator with dense domain. So is $A^{n}$ for every positive $n$. Indeed $D\left(A^{\infty}\right)=\bigcap_{n} D\left(A^{n}\right)$ is dense in $X$. The spectrum of $A$, denoted $\sigma[A]$, is contained in the halfplane $\operatorname{Re} \lambda \leqq w^{+}$.

Next let $\delta(w)$ be the class of all countably additive set functions $\alpha$ defined on the bounded Borel sets of $[0, \infty)$, such that

$$
\int_{0}^{\infty}|| T(\xi)|| d_{\xi}|\alpha|<\infty
$$

where $|\alpha|$ is the variation of $\alpha$. If the integral above is taken as the norm $\|\alpha\|$ of $\alpha, \delta(w)$ becomes a $B$-space, indeed a $B$-algebra (commutative, with unit) if the product of two elements is defined as their convolution. This algebra was introauced by Phillips $[3 ; 4]$ and is basic to our theory. We collect here only such properties of this algebra as we shall need, and refer 
the reader for more information to the papers cited. We denote by $L(w)$ the closed subspace (also an ideal) of those elements which are absolutely continuous with respect to Lesbesgue measure. For use in spectral theory, we distinguish two classes of maximal ideals in $\delta(w)$; the class $U$ consisting of those maximal ideals which contain $L(w)$, and the class $W$ of the remaining maximal ideals. There is a $1: 1$ correspondence between the elements of $W$ and the complex numbers $\lambda, \operatorname{Re} \lambda \leqq w^{+}$, exemplified by the relation:

$$
\alpha(m)=\int_{0}^{\infty} e^{\lambda \xi} d_{\xi} \alpha, \quad \alpha \in S(w), m \in W, \text { and } m \sim \lambda .
$$

We denote by $\phi(\lambda ; \alpha)$ the Laplace-Stieltjes transform of an element $\alpha \in \mathcal{S}(w)$ :

$$
\phi(\lambda ; \alpha)=\int_{0}^{\infty} e^{\lambda \xi} d_{\xi} \alpha
$$

in its halfplane of absolute convergence $\operatorname{Re} \lambda \leqq w^{+}$. Since by (iii) $w^{+}>-\infty$, by Lerch's theorem $\phi(\lambda ; \alpha)$ completely characterises $\alpha$.

The Hille-Phillips calculus. If for any $\alpha \in S(w)$ we define

$$
\theta(\alpha) x=\int_{0}^{\infty} T(\xi) x d_{\xi} \alpha, \quad x \in X
$$

then $\theta(\alpha)$ is a linear bounded transformation over $X,\|\theta(\alpha)\| \leqq\|\alpha\|$, and $\theta$ moreover defines a homomorphism from $\delta(w)$ into $E(X)$, the algebra of endomorphisms over $X$. Let $R$ be the second commutant of the set $\{T(\xi), \xi>0\}$. Then $R$ is a commutative $B$-algebra with unit and $\theta$ takes $\mathcal{S}(w)$ into $R$. Consequently, $\theta$ induces a corresponding homomorphism on the maximal ideals of $R$ into those of $S(w)$, and via the Gelfand theory, affords a means to study the spectral properties of the operators $\theta(\alpha)$. Let $W^{\prime}$ be the maximal ideals of $R$ which map into $W$, and $U^{\prime}$ those that map into $U$. Then for every $m^{\prime} \in W^{\prime}$ there is thus a complex number $\lambda, \operatorname{Re} \lambda \leqq w^{+}$, and

$$
\theta(\alpha)\left(m^{\prime}\right)=\phi(\lambda ; \alpha), \quad a\left(m^{\prime}\right)=\lambda,
$$

and for $m^{\prime} \in U^{\prime}$, if $\alpha \in L(w)$,

$$
\theta(\alpha)\left(m^{\prime}\right)=0
$$

Moreover:

$$
\sigma\left[A^{n}\right]=\left\{a\left(m^{\prime}\right)^{n}, m^{\prime} \in W^{\prime}\right\} .
$$

The transformation (2.2) provides the basic construction of the HillePhillips calculus. Thus let $S(\xi)$ be any strongly continuous semigroup [satisfying $H$ (i) and (ii)] over a possibly different $B$-space (although we shall continue to denote it by $X)$. We say that $S(\xi)$ is majorized by $T(\xi)$ if for every $\xi>0$,

$$
\|S(\xi)\| \leqq M\|T(\xi)\|, \quad M<\infty
$$


It is evident that in the definition (2.2) we can replace $T(\xi)$ by $S(\xi)$. If we denote the infinitesimal generator of $S(\xi)$ by $B$, Hille [1] defines for $\alpha \in \mathcal{S}(w)$ :

$$
f(B) x=\int_{0}^{\infty} S(\xi) x d_{\xi \alpha}
$$

where

$$
f(\lambda)=\phi(\lambda ; \alpha)
$$

If in particular,

$$
S(\xi)=I \exp \lambda \xi, \quad \operatorname{Re} \lambda \leqq w^{+},
$$

(2.3) reduces to

$$
f(\lambda I) x=f(\lambda) x, \text { or, } f(\lambda I)=I f(\lambda),
$$

and is thus a consistent operational extension.

$\boldsymbol{S}(w)$ as an Operator Algebra over $L(w)$. Since $L(w)$ is an ideal in $\mathfrak{S}(w)$, each element of $S(w)$ can also be considered as a linear operator over $L(w)$ if we define, for any $\alpha \in \mathcal{S}(w)$ and every $f \in L(w)$,

$$
T_{\alpha} f=\alpha * f
$$

where the asterisk denotes convolution. We shall denote the operator norm by $\left\|T_{\alpha}\right\|,\left\|T_{\alpha}\right\|=\operatorname{Sup}\|\alpha * f\| /\|f\|$. If for every $\xi>0$ we define a set function $E_{\xi}$ such that for any bounded Borel set $B$ of $[0, \infty)$,

$$
E_{\xi}(B)=1 \text { if } \xi \in B \text {, and } 0 \text { otherwise, }
$$

$E_{\xi} \in \delta(w),\left\|E_{\xi}\right\|=\|T(\xi)\|$, and $\theta\left(E_{\xi}\right)=T(\xi)$. Further, considered as operators over $L(w)$, they form a semigroup satisfying $(\mathrm{H})$. This is of course a semigroup of translation operators over $L(w)$ :

$$
\left(E_{\xi} * f\right)(t)=f(t-\xi),
$$

If in (2.2) we put $T(\xi) \equiv E_{\xi}, X=L(w), x \sim f$, we obtain

$$
\int_{0}^{\infty} E_{\xi} * f d_{\xi} \alpha
$$

which is simply $\alpha * f$. Let $A_{w}$ denote the infinitesimal generator of the semigroup $E_{\xi}$. Then $D\left(A_{w}\right)$ consists precisely of those $f$ such that $f(0)=0, f, f^{\prime}$ $\in L(w)$, and $A_{w} f=-f^{\prime}$. If $f \in D\left(A_{w}^{k}\right), k$ being a positive integer, we have, since $\theta$ is a continuous homomorphism that for every $x \in X$

$$
\begin{aligned}
\theta\left(A_{w} f\right) x & =\operatorname{limit}_{\xi \rightarrow 0} \xi^{-1} \theta\left(E_{\xi} * f-f\right) x \\
& =\operatorname{limit}_{\xi \rightarrow 0} \xi^{-1}[T(\xi) x-x] \\
& =A \theta(f) x
\end{aligned}
$$


and hence readily that

$$
\theta\left(A_{w}^{n} f\right) x=A^{n} \theta(f) x, \quad n=1,2, \cdots k ; x \in X .
$$

Setting $T(\xi)=I \exp \lambda \xi$, this specializes to

$$
\lambda^{n} \phi(\lambda ; f)=\phi\left(\lambda ; A_{w}^{n} f\right), \quad n=1,2, \cdots, k .
$$

We conclude this introductory section with a Lemma of a general nature we shall need:

Lemma 2.1. Let $C$ be a closed linear operator with domain and range in $X$. Let $x \in D(C)$, and further let

$$
T(\xi) C x=C T(\xi) x \quad \text { for every } \xi>0 .
$$

Then for every $\alpha \in \$(w), \theta(\alpha) x \in D(C)$ and

$$
\theta(\alpha) C x=C \theta(\alpha) x .
$$

Proof.

$$
\theta(\alpha) C x=\int_{0}^{\infty} T(\xi) C x d_{\xi \alpha}=\int_{0}^{\infty} C T(\xi) x d_{\xi \alpha}
$$

and since $C$ is closed linear, we have readily,

$$
\int_{0}^{\infty} C T(\xi) x d_{\xi} \alpha=C \int_{0}^{\infty} T(\xi) x d_{\xi \alpha}=C \theta(\alpha) x
$$

proving the lemma.

3. The extended calculus. The Hille-Phillips calculus, as we have seen, yields only bounded operators. The extension of this calculus to unbounded operators requires first that the function class $f(\lambda)$ be suitably enlarged. To do this we begin with the notion of a multiplier. Let $\varphi(\lambda)$ be any Lesbesgue measurable function of $\lambda, \operatorname{Re} \lambda \leqq w^{+}$. We associate with it an operator $C_{w}$ with domain and range in $L(w)$ as follows:

$$
f \in D\left(C_{w}\right) \quad \text { and } \quad C_{w} f=g
$$

if and only if

$$
\varphi(\lambda) \phi(\lambda ; f)=\phi(\lambda ; g), \quad f, g \in L(w) .
$$

That the operator is well-defined follows from the uniqueness theorem for Laplace transforms, since $w^{+}>-\infty$. Since $\varphi(\lambda)$ is a multiplier it is immediate that $C_{w}$ is a closed linear operator with nonempty domain. Moreover, $D\left(C_{w}\right)$ is an ideal in $\mathcal{S}(w)$ :

Lemma 3.1. Let $\varphi(\lambda) \sim C_{w}$ and let $f \in D\left(C_{w}\right)$. Then for any $\alpha \in \mathcal{S}(w), \alpha * f$ $\in D\left(C_{w}\right)$ and 


$$
C_{w}(\alpha * f)=\left(C_{w} f\right) * \alpha .
$$

The factor theorem $(1$, p. 362$)$ has an immediate generalization in this setting:

THEOREM 3.1. Let $\varphi(\lambda) \sim C_{w}$. Then a necessary and sufficient condition that

$$
\varphi(\lambda)=\phi(\lambda ; \alpha) \text { for some } \alpha \in \delta(w)
$$

is that

$$
D\left(C_{w}\right)=L(w) .
$$

Proof. See [5] if necessary.

Corollary. Let $\varphi(\lambda) \sim C_{w}$. If $\mu \in \rho\left(C_{w}\right)$ (Resolvent set of $C_{w}$ ), then there is an $\alpha \in S(w)$ such that $R\left(\mu ; C_{w}\right)=T_{\alpha}$, and

$$
\phi(\lambda ; \alpha)=(\mu-\varphi(\lambda))^{-1} .
$$

Proof. Since for every $f \in L(w)$

$$
[\mu-\varphi(\lambda)]_{\phi}\left(\lambda ; R\left(\mu ; C_{w}\right) f\right)=\phi(\lambda ; f)
$$

it follows readily that $(\mu-\varphi(\lambda))^{-1}$ is a multiplier whose domain is $L(w)$. The theorem above thus applies and the corollary is immediate.

We may note in passing that Lemma 3.1 characterizes the operators $C_{w}$ in the sense that if any closed linear operator $C$ with domain and range in $L(w)$ has the property

$$
C\left(E_{\xi} * f\right)=E_{\xi} * C f \quad \text { for every } \xi>0,
$$

for every $f$ in its domain, then there is a multiplier $\varphi(\lambda)$ such that $\varphi(\lambda) \sim C_{w}$ $=C$.

We know as yet no way of extending an arbitrary multiplier $\varphi(\lambda)$ to a corresponding operator $\varphi(A)$ over $X$. Fortunately, for the semigroup theoretic applications we have in mind, this generality is unnecessary. From this point on, we accordingly confine our study to those $C_{w}$ with the property that $D\left(C_{w}\right)$ is dense in $L(w)$, and denote this class by $\mathfrak{T}$. We begin with two results which provide essentially the basis for the extension of the Hille-Phillips calculus.

We shall say that a sequence $\left\{\alpha_{n}\right\} \in \mathcal{S}(w)$ approximates the identity strongly (over $L(w)$ ) if for every $f \in L(w),\left\|\alpha_{n} * f-f\right\| \rightarrow 0$.

THEOREM 3.2. Let $\left\{\alpha_{n}\right\}$ be a sequence approximating the identity strongly over $L(w)$. Then $\theta\left(\alpha_{n}\right)$ approximates the identity strongly over $X$, i.e., $\left\|\theta\left(\alpha_{n}\right) x-x\right\|$ $\rightarrow 0$, for every $x \in X$.

Proof. A proof has been given by Phillips [3]. Here we give a different and self-contained proof, obtaining en route some results which we shall use later on again. We need two lemmas. 
Lemma 3.2. We can find a sequence $\left\{g_{n}\right\} \in D\left(A_{w}^{\infty}\right)$ such that

(i) $g_{n}$ approximates the identity strongly over $L(w)$ and $\theta\left(g_{n}\right)$ over $X$,

(ii) $\left\|g_{n}\right\| \rightarrow \gamma, 1 \leqq \gamma<\infty$.

Proof. Let $\left\{f_{n}\right\}$ be a sequence of Lévy stable densities [see [6]] such that

$$
\phi\left(\lambda ; f_{n}\right)=\operatorname{Exp}-(-\lambda)^{\zeta / n}, \quad 0<\zeta<1, \operatorname{Re} \lambda \leqq 0 .
$$

Let $\sigma>w^{+}$and set

$$
g_{n}(\xi)=f_{n}(\xi) \operatorname{Exp}-\sigma \xi, \quad \xi \geqq 0 .
$$

Then clearly $g_{n}(\cdot) \in L(w)$, actually to $D\left(A_{w}^{\infty}\right)$. Further for $x \in X$,

$$
\left\|\theta\left(g_{n}\right) x-x\right\| \leqq \int_{0}^{\infty} e^{-\sigma \xi} f_{n}(\xi)\|T(\xi) x-x\| d \xi+\|x\|\left|\phi\left(-\sigma ; f_{n}\right)-1\right| .
$$

Since $e^{-\sigma \xi}\|T(\xi) x-x\|$ is a bounded continuous function, the first term goes to zero by the weak convergence property of the densities $\left\{f_{n}\right\}$, and the second term obviously goes to zero. Hence $\theta\left(g_{n}\right)$ approximates the identity strongly. In particular, we see that $g_{n}$ approximates the identity strongly over $L(w)$. To prove (ii), we note that

$$
\left\|g_{n}\right\|=\int_{0}^{\infty}\left\|T\left(\xi t_{n}\right)\right\| e^{-\sigma \xi t_{n}} f_{1}(\xi) d \xi
$$

where $t_{n}=(+1 / n)^{1 / 5}$. Clearly, the sequence $\left\{\left\|g_{n}\right\|_{\}}\right\}$is bounded. If $\|T(\xi)\|$ is continuous at the origin, $\left\|g_{n}\right\| \rightarrow 1$. Otherwise, we can choose a convergent subsequence, and in any case:

$$
1 \leqq \liminf _{\xi \rightarrow 0}\|T(\xi)\| \leqq \gamma \leqq \lim \sup \|T(\xi)\| .
$$

Lemma 3.3. Let $\alpha \in \mathcal{S}(w)$. Then

$$
\|\theta(\alpha)\| \leqq \gamma\left\|T_{\alpha}\right\|
$$

Proof. Let $x \in X,\|x\|=1$. Then using Lemma 3.2,

$$
\begin{aligned}
\|\theta(\alpha) x\| & =\lim \left\|\theta(\alpha) \theta\left(g_{n}\right) x\right\| \\
& \leqq \lim \sup \left\|\alpha * g_{n}\right\| \leqq \lim \sup \left\|T_{\alpha}\right\|\left\|g_{n}\right\| \\
& =\gamma\left\|T_{\alpha}\right\| .
\end{aligned}
$$

Hence $\|\theta(\alpha)\| \leqq \gamma\left\|T_{\alpha}\right\|$.

The theorem follows easily from the lemmas. Since $\left\{\alpha_{n}\right\}$ converges strongly, $\left\|T_{\alpha_{n}}\right\|$ is bounded, and by Lemma 3.3, so is $\left\|\theta\left(\alpha_{n}\right)\right\|$. For any $f \in L(w)$ and $x \in X$,

$$
\left\|\theta\left(\alpha_{n}\right) \theta(f) x-\theta(f) x\right\| \leqq\left\|\alpha_{n} * f-f\right\|\|x\| \rightarrow 0
$$


Since it follows in particular from Lemma 3.2 that the elements of the form $\theta(f) x$ are dense in $X$, an application of the Banach-Steinhaus theorem [1, p. 25] yields the desired result.

Theоrem 3.3. Let $D$ be a dense subset of $L(w)$. Then we can find a sequence $\left\{f_{n}\right\}$ in $D$ such that:

(i) $f_{n}$ approximates the identity strongly,

(ii) $\left\|f_{n}\right\| \rightarrow \gamma$.

Proof. Let $\left\{h_{n}\right\}$ be any sequence in $L(w)$ approximating the identity strongly. Since $D$ is dense in $L(w)$, we can find $\left\{f_{n}\right\}$ in $D$ such that

$$
\left\|h_{n}-f_{n}\right\| \leqq 1 / n
$$

and this implies that the sequence $\left\{f_{n}\right\}$ approximates the identity strongly as well. (This observation is due to R. S. Phillips and replaces a lengthier argument of the author's.) In particular, we may choose the sequence $\left\{g_{n}\right\}$ defined by (3.2) in place of $\left\{h_{n}\right\}$, and since $\left\|g_{n}\right\| \rightarrow \gamma$, it follows that (ii) can be satisfied as well.

COROLlary. The intersection of two dense ideals in $L(w)$ is dense.

Proof. Let $\left\{f_{n}\right\},\left\{g_{n}\right\}$ be sequences approximating the identity in two dense ideals $D_{1}$ and $D_{2}$ respectively. Then the sequence $\left\{f_{n} * g_{n}\right\} \in D_{1} \cap D_{2}$ and approximates the identity as well. Hence $D_{1} \cap D_{2}$ is dense in $L(w)$.

Since by definition $D\left(C_{w}\right)$, for any $\varphi(\lambda) \in \mathscr{T}, \varphi(\lambda) \sim C_{w}$, is a dense ideal, it follows from the theorem and corollary just proved that we can find a sequence in $D\left(C_{w}\right) \cap D\left(A_{w}^{\infty}\right)$ approximating the identity strongly.

We next show that the class $\mathscr{M}$ is an algebra.

Theorem 3.4. Let $\varphi_{1}(\lambda), \varphi_{2}(\lambda) \in \mathfrak{T}$. If $\mu_{1}, \mu_{2}$ are any two complex numbers, $\mu_{1} \varphi_{1}(\lambda)+\mu_{2} \varphi_{2}(\lambda) \in \mathfrak{T}$. The product $\varphi_{1}(\lambda) \varphi_{2}(\lambda) \in \mathfrak{T l}$ also.

Proof. Let $\varphi_{1}(\lambda) \sim C_{1}, \varphi_{2}(\lambda) \sim C_{2}$ and $\varphi_{1}(\lambda) \varphi_{2}(\lambda) \sim C_{3}$. Clearly $\mu_{1} \varphi_{1}(\lambda)$ $+\mu_{2} \varphi_{2}(\lambda)$ is a multiplier, and since its domain certainly includes $D\left(C_{1}\right) \cap D\left(C_{2}\right)$ which is dense by the corollary to Theorem 3.3 , it belongs to $\mathfrak{\text { Tr }}$. Let $\left\{g_{n}\right\}$, $\left\{h_{n}\right\}$ be sequences approximating the identity in $D\left(C_{1}\right)$ and $D\left(C_{2}\right)$ respectively. Then the sequence $\left\{g_{n} * h_{n}\right\}$ approximates the identity and belongs to $D\left(C_{3}\right)$ as well. Hence $\varphi_{1}(\lambda) \varphi_{2}(\lambda) \in \mathscr{M}$ also.

Any multiplier $\varphi(\lambda) \in \mathscr{N}$ is analytic in $\operatorname{Re} \lambda<w^{+}$, continuous in $\operatorname{Re} \lambda \leqq w^{+}$. This follows readily from the fact that it has a dense domain. Moreover, the correspondence $\varphi(\lambda) \sim C_{w}$ is $1: 1$ for any $\varphi(\lambda) \in 9$.

The class $\mathfrak{T}$ certainly includes all polynomials in $\lambda$. Actually, the polynomials belong to an important subclass of $\mathfrak{T}$, namely the subclass of multipliers whose domains include $D\left(A_{w}^{\infty}\right)$. This subclass, which we shall denote by $\mathfrak{M}_{\infty}$, is easily verified to be a subalgebra of $\mathfrak{T}$, since a multiplier $\in \mathfrak{M}_{\infty}$ takes $D\left(A_{w}^{\infty}\right)$ into itself. This is a proper subclass, however, as the following example shows. Let 


$$
\varphi(\lambda)=\operatorname{Exp}(\sigma-\lambda)^{\beta}, \quad 0<\beta<1, \sigma>w^{+}
$$

where $z^{\beta}$ is taken positive for $z$ positive. Then if $\beta<\zeta$, the sequence $\left\{g_{n}\right\}$ defined by (3.2) certainly belongs to its domain, so that this is a multiplier in $\mathbb{T}$. On the other hand, the clement $g \in D\left(A_{w}\right)$ where

$$
\phi(\lambda ; g)=\operatorname{Exp}-(\sigma-\lambda)^{\eta}, \quad 0<\eta<\beta
$$

does not belong to its domain.

Construction of the operator extensions. Given any $\varphi(\lambda) \in \mathscr{M}$, we shall now describe how an operator extension $\varphi(A)$ may be obtained as a closed linear operator with dense domain. First let $f, g \in D\left(C_{w}\right), C_{w} \sim \varphi(\lambda) \in \mathfrak{N}$. Suppose that for some $x, y \in X, \theta(f) x=\theta(g) y$. Let $\left\{h_{n}\right\}$ be a sequence approximating the identity in $D\left(C_{w}\right)$. Then using Lemma 3.1,

$$
\begin{aligned}
\theta\left(h_{n}\right) \theta\left(C_{w} f\right) x & =\theta\left(h_{n} * C_{w} f\right) x \\
& =\theta\left(C_{w} h_{n}\right) \theta(f) x \\
& =\theta\left(C_{w} h_{n}\right) \theta(g) y \\
& =\theta\left(h_{n}\right) \theta\left(C_{w} g\right) y .
\end{aligned}
$$

Using Theorem 3.2 and taking limits we obtain:

$$
\theta\left(C_{w} f\right) x=\theta\left(C_{w} g\right) y .
$$

Analogous to (2.4) and (2.5) we can therefore define an operator $C_{0}$ with domain and range in $X$ as follows:

$$
\begin{aligned}
D\left(C_{0}\right) & \equiv \text { Elements of the form } \theta(f) x, f \in D\left(C_{w}\right) \text { and } x \in X ; \\
C_{0} \theta(f) x & =\theta\left(C_{w} f\right) x .
\end{aligned}
$$

Thus defined, the operator $C_{0}$ is not necessarily linear or closed, but is readily extended to be so. We recall that an operator $U$ (linear or not) is said to be closed if whenever $x_{n} \in D(U), x_{n} \rightarrow x$ and $U x_{n}$ converges, $x \in D(U)$ and $U x=$ limit $U x_{n}$. An operator $U$ is said to have a closed linear extension if there is an operator $C$ which is closed, $D(C) \supset D(U)$ and $C x=U x$ for $x \in D(U)$. In this case the smallest closed extension of $U$ has an obvious definition, and we denote the latter by $[U]_{c}$.

Theorem 3.5. Let $C_{w} \sim \varphi(\lambda) \in \mathfrak{M}$. Then the operator $C_{0}$ defined by (3.3) can be extended to be closed. Let $C$ denote the smallest closed extension of $C_{0}$. Then $C$ is closed linear, and $D(C)$ contains a dense sub-domain of $D\left(A^{\infty}\right)$. For any $T \in E(X)$ which commutes with $T(\xi)$ for every $\xi>0$,

$$
T C x=C T x \quad \text { for } x \in D(C) .
$$

Proof. We begin by showing that $C_{0}$ can be extended to be closed. For this, let $\left\{x_{n}\right\},\left\{y_{n}\right\}$ be two sequences in $D\left(C_{0}\right)$ both converging to the same element $x$, and suppose $C_{0} x_{n}$ and $C_{0} y_{n}$ are also convergent with limits $y$ and $z$ 
respectively. To see then that $y$ and $z$ must be equal, we may use a sequence $\left\{h_{n}\right\}$ approximating the identity in $D\left(C_{w}\right)$. We have, using Lemma 3.1,

$$
\theta\left(h_{m}\right) C_{0} x_{n}=\theta\left(C_{w} h_{m}\right) x_{n}
$$

for every $n$, so that taking limits,

$$
\theta\left(h_{m}\right) y=\theta\left(C_{w} h_{m}\right) x .
$$

Similarly,

$$
\theta\left(h_{m}\right) z=\theta\left(C_{w} h_{m}\right) x .
$$

Hence $\theta\left(h_{m}\right) y=\theta\left(h_{m}\right) z$, and using Theorem 3.2 and taking limits in $m$ we get $y=z$. Hence $C_{0}$ can be extended to be closed by closing the graph of $C_{0}$ (see [1] for the notion of graph). More explicitly, we define an operator $C$ with domain and range in $X$ by:

$x \in D(C)$ if and only if there is a sequence $\left\{x_{n}\right\}$ in $D\left(C_{0}\right), x_{n} \rightarrow x$, and

$$
C_{0} x_{n} \text { converges; and }
$$

$$
C x=\operatorname{limit} C_{0} x_{n} .
$$

It is evident that $C$ is closed since the graph of $C$ is closed, and is indeed the smallest closed extension of $C_{0}$. The latter property leads easily to (3.4). For, if $x \in D(C)$, we have by definition that $C x=\operatorname{limit} C_{0} x_{n}, x_{n} \rightarrow x$. Now as in (3.5),

$$
\theta\left(h_{m}\right) C_{0} x_{n}=\theta\left(C_{w} h_{m}\right) x_{n}=C_{0} \theta\left(h_{m}\right) x_{n}
$$

so that by passage to the limit in $n$ we have

$$
\theta\left(h_{m}\right) C x=\theta\left(C_{w} h_{m}\right) x=C_{0} \theta\left(h_{m}\right) x
$$

and hence

$$
C x=\operatorname{limit} \theta\left(C_{w} h_{m}\right) x=\operatorname{limit} C_{0} \theta\left(h_{m}\right) x .
$$

If $T$ is as in the hypothesis, we have first from Lemma 2.1 trivially that $T$ commutes with $\theta\left(h_{m}\right)$ for every $m$, and for $x \in D(C)$ therefore

$$
\begin{aligned}
T C x=\operatorname{limit} T \theta\left(C_{w} h_{m}\right) x & =\operatorname{limit} \theta\left(C_{w} h_{m}\right) T x \\
& =\operatorname{limit} C_{0} \theta\left(h_{m}\right) T x
\end{aligned}
$$

and since $C$ is closed we obtain

$$
T C x=C T x .
$$

To show that $C$ is linear, let $x, y \in D(\mathcal{C})$. Then

$$
\begin{aligned}
C_{0} \theta\left(h_{m}\right)(x+y) & =C_{0} \theta\left(h_{m}\right) x+C_{0} \theta\left(h_{m}\right) y \\
& =\theta\left(h_{m}\right) C x+\theta\left(h_{m}\right) C y
\end{aligned}
$$


where we have used (3.4). Passing to the limit in $m$ we have:

$$
C(x+y)=C x+C y .
$$

That $\lambda C x=C \lambda x$ for any complex number follows trivially from (3.4). Hence $C$ is linear. Finally, since the sequence $\left\{h_{n}\right\}$ can be chosen in $D\left(C_{w}\right) \cap D\left(A_{w}^{\infty}\right)$ and elements of the form $\theta\left(h_{m}\right) x$ belong to $D\left(A^{\infty}\right)$ (cf. (2.4)), it follows that $D(C)$ contains a dense subdomain of $D\left(A^{\infty}\right)$.

We have thus the basic construction of the extended calculus:

Definition 3.1. Let $\varphi(\lambda) \in \mathscr{T}$, and let $\varphi(\lambda) \sim C_{w}$. The operator extension $\varphi(A)$ is then defined to be the closed linear operator $C$ given by (3.6). More generally, if $B$ is the infinitesimal generator of any strongly continuous semigroup $S(\xi)$ majorized by $T(\xi)$, we again define $\varphi(B)$ as the operator $C$ given by (3.6) except that now in the definition of $\theta$ given by (2.2) we replace $T(\xi)$ by $S(\xi)$.

Some consistency properties of these operator extensions are next in order. First let $\varphi(\lambda)=\phi(\lambda ; \alpha), \alpha \in \mathcal{S}(w)$. Then clearly $\varphi(\lambda) \in \mathscr{N}$ and has $L(w)$ as its domain. Further if $f \in L(w), C_{w} \sim \varphi(\lambda)$,

$$
\theta\left(C_{w} f\right) x=C_{0} \theta(f) x=\theta(\alpha * f) x=\theta(\alpha) \theta(f) x
$$

so that the smallest closed extension of $C_{0}$ is $\theta(\alpha)$. Hence Definition 3.1 is consistent with the Hille-Phillips calculus. Again, if we take $S(\xi)=I \exp \lambda \xi$, $\operatorname{Re} \lambda \leqq w^{+}$, we have readily that $\varphi(\lambda I)=I \varphi(\lambda)$. If we take $S(\xi) \equiv E_{\xi}, X=L(w)$ we obtain $\varphi\left(A_{w}\right)=C_{w}$. Finally let $\varphi(\lambda)$ be any polynomial in $\lambda$, say $\varphi(\lambda)$ $=\sum_{0}^{n} a_{k} \lambda^{k}, a_{n} \neq 0$. We shall now show that $\varphi(A)=\sum_{0}^{n} a_{k} A^{k}$. We note first that for any $x \in X$, using (2.4) and (2.5):

$$
\theta\left(C_{w} h_{n}\right) x=\sum_{0}^{n} a_{k} A^{k} \theta\left(h_{n}\right) x
$$

where since $D\left(C_{w}\right)$ obviously contains $D\left(A_{w}^{n}\right),\left\{h_{n}\right\}$ is a sequence in $D\left(A_{w}^{n}\right)$ approximating the identity strongly. For $x \in D\left(A^{n}\right)$ on the other hand, by Lemma 2.1 , since $A^{k}$ is closed,

$$
\sum_{0}^{n} a_{k} A^{k} \theta\left(h_{n}\right) x=\theta\left(h_{n}\right) \sum_{0}^{n} a_{k} A^{k} x .
$$

Hence taking limits in (3.8) we have:

$$
\varphi(A) x=\sum_{0}^{n} a_{k} A^{k} x, \quad x \in D\left(A^{n}\right) .
$$

Again for $x \in D(\varphi(A))$, by (3.7)

$$
\varphi(A) x=\operatorname{limit} \theta\left(C_{w} h_{n}\right) x=\operatorname{limit} \sum_{0}^{n} a_{k} A^{k} \theta\left(h_{n}\right) x .
$$


Since $\sum_{0}^{n} a_{k} A^{k}$ is closed, it follows that

$$
\varphi(A) x=\sum_{0}^{n} a_{k} A^{k} x, \quad x \in D(\varphi(A)) .
$$

Hence $\varphi(A)=\sum_{0}^{n} a_{k} A^{k}$.

When $A \in E(X), \varphi(A) \in E(X)$. Thus:

Theorem 3.6. Let $A \in E(X)$. Let $\varphi(\lambda) \in \mathfrak{T}$. Then $\varphi(A) \in R$.

Proof. Since $A \in E(X)$, for any $\alpha \in S(w)$ we have:

$$
\theta(\alpha)=\int_{0}^{\infty} e^{A \xi} d \xi \alpha .
$$

Moreover, from the Gelfand theory,

$$
\sigma[\theta(\alpha)]=\int_{0}^{\infty} \operatorname{Exp} \sigma[A] \xi d_{\xi} \alpha .
$$

In particular, if $\left\{h_{n}\right\}$ is a sequence in $D\left(\varphi\left(A_{w}\right)\right)$ approximating the identity strongly,

$$
\sigma\left[\theta\left(h_{n}\right)\right]=\int_{0}^{\infty} h_{n}(\xi) \operatorname{Exp} \sigma[A] \xi d \xi
$$

Again since $A \in E(X), \sigma[A]$ is a compact subset of $\operatorname{Re} \lambda \leqq w^{+}$, and since $\phi\left(\lambda ; h_{n}\right) \rightarrow 1$ uniformly in each compact subset of $\operatorname{Re} \lambda \leqq w^{+}$, it follows that we can find $N$ such that $\phi\left(\lambda ; h_{N}\right)$ is bounded away from zero for $\lambda \in \sigma[A]$, or $\theta\left(h_{N}\right)$ has an inverse in $R$. Hence for any $x \in X$, there is a $y \in X$, such that $\theta\left(h_{N}\right) y=x$, so that $x \in D(\varphi(A))$. By the closed graph theorem [1, p. 30], $\varphi(A) \in E(X)$. From (3.4) it follows that $\varphi(A) \in R$.

We turn finally to the algebraic properties of the operators $\varphi(A)$.

TheOREM 3.7. Let $\varphi_{1}(\lambda), \varphi_{2}(\lambda) \in \mathscr{T}$. Then letting

$$
\begin{aligned}
& \varphi_{3}(\lambda)=\varphi_{1}(\lambda)+\varphi_{2}(\lambda), \\
& \varphi_{4}(\lambda)=\varphi_{1}(\lambda) \varphi_{2}(\lambda)
\end{aligned}
$$

we have

$$
\begin{aligned}
\varphi_{3}(A) & =\left[\varphi_{1}(A)+\varphi_{2}(A)\right]_{c}, \\
\varphi_{4}(A) & =\left[\varphi_{1}(A) \cdot \varphi_{2}(A)\right]_{c} .
\end{aligned}
$$

Proof. Let $\left\{h_{n}\right\}$ be a sequence in the intersection of the domains $\varphi_{1}\left(A_{w}\right)$ and $\varphi_{2}\left(A_{w}\right)$. For $x \in D\left(\varphi_{1}(A)\right) \cap D\left(\varphi_{2}(A)\right)$ we have then using (3.7)

$$
\varphi_{1}(A) x+\varphi_{2}(A) x=\operatorname{limit}\left[\varphi_{1}(A)+\varphi_{2}(A)\right] \theta\left(h_{n}\right) x .
$$

By Theorem 3.4, $\varphi_{3}(\lambda) \in \mathscr{T}$, and further 


$$
\left[\varphi_{1}(A)+\varphi_{2}(A)\right] \theta\left(h_{n}\right) x=\varphi_{3}(A) \theta\left(h_{n}\right) x .
$$

Hence taking limits and noting that $\varphi_{3}(A)$ is closed:

$$
\left[\varphi_{1}(A)+\varphi_{2}(A)\right] x=\varphi_{3}(A) x .
$$

Hence $\left[\varphi_{1}(A)+\varphi_{2}(A)\right]$ can be extended to be closed. Further if $x \in D\left(\varphi_{3}(A)\right)$, using (3.7) again

$$
\begin{aligned}
\varphi_{3}(A) x & =\operatorname{limit} \varphi_{3}(A) \theta\left(h_{n}\right) x \\
& =\operatorname{limit}\left[\varphi_{1}(A)+\varphi_{2}(A)\right] \theta\left(h_{n}\right) x \\
& =\left[\left[\varphi_{1}(A)+\varphi_{2}(A)\right]\right]_{c} x .
\end{aligned}
$$

Hence (3.9) follows. (3.10) can be established similarly.

We may note that if we define sum and product by (3.9) and (3.10) the class of operators $\varphi(A)$ for each $A$ can be made into a commutative algebra which contains $\theta(\delta(w))$ as a subalgebra. We can obtain a spectral theory for this algebra which parallels that for $\theta(\mathcal{S}(w))$ given by Hille [1] and Phillips [4].

4. Spectral theory. In this section we study the spectral properties of the operators $\varphi(A)$. We begin with a result which has been proved in a special case by Phillips [3] by a different method.

TheOREM 4.1. Let $\varphi(\lambda) \in \mathfrak{T l}$. If

$$
\mu \in \rho\left(\varphi\left(A_{w}\right)\right)
$$

then

$$
\mu \in \rho(\varphi(A))
$$

and

$$
R(\mu ; \varphi(A))=\theta\left(R\left(\mu ; \varphi\left(A_{w}\right)\right) .\right.
$$

Proof. Let $\left\{h_{n}\right\}$ be a sequence in $D\left(\varphi\left(A_{w}\right)\right)$ approximating the identity strongly. Then

$$
\begin{aligned}
& {\left[\mu E_{0}-\varphi\left(A_{w}\right)\right] R\left(\mu ; \varphi\left(A_{w}\right)\right) h_{n}=h_{n},} \\
& R\left(\mu ; \varphi\left(A_{w}\right)\right)\left[\mu E_{0}-\varphi\left(A_{w}\right)\right] h_{n}=h_{n} .
\end{aligned}
$$

For any $x \in X$, using the Corollary to Theorem 3.1,

$$
\begin{aligned}
{[\mu I-\varphi(A)] \theta\left(R\left(\mu ; \varphi\left(A_{w}\right)\right)\right.} & \theta\left(h_{n}\right) x \\
& =\theta\left(h_{n}\right) x=\theta\left(R\left(\mu ; \varphi\left(A_{w}\right)\right)[\mu I-\varphi(A)] \theta\left(h_{n}\right) x .\right.
\end{aligned}
$$

Using the first part of this equality and taking limits in $n$, we obtain since $\varphi(A)$ is closed:

$$
[\mu I-\varphi(A)] \theta\left(R\left(\mu ; \varphi\left(A_{w}\right)\right) x=x\right.
$$


If $x \in D(\varphi(A))$, we have from (3.7):

$$
\varphi(A) x=\operatorname{limit} \varphi(A) \theta\left(h_{n}\right) x
$$

and the second half of the equality in (4.2) readily yields

$$
x=\theta\left(R\left(\mu ; \varphi\left(A_{w}\right)\right)[\mu I-\varphi(A)] x\right.
$$

thus establishing (4.1).

We next prove a spectral mapping theorem analogous to the one proved by Hille [1, p. 313]. For this purpose it is convenient to use the Gelfand theory for the $B$-algebra $R$ as developed by Phillips [4] (cf. §2).

Theorem 4.2. Let $\varphi(\lambda) \in \Re$. Then

$$
\sigma[\varphi(A)] \supset \varphi(\sigma[A]) .
$$

If for some $\mu \in \rho\left[\varphi\left(A_{w}\right)\right]$,

$$
R\left(\mu ; \varphi\left(A_{w}\right)\right) \in L(w)
$$

then

$$
\sigma[\varphi(A)]=\varphi(\sigma[A]) .
$$

Proof. We begin by showing that if $\mu \in \rho[\varphi(A)]$, then necessarily, $R(\mu ; \varphi(A)) \in R$. For if $T \in E(X)$ and commutes with $T(\xi)$ for every $\xi>0$, we have from (3.4)

$$
T \varphi(A) x=\varphi(A) T x, \quad x \in D(\varphi(A)) .
$$

Hence for any $x \in X$,

$$
\begin{aligned}
R(\mu ; \varphi(A)) T x & =R(\mu ; \varphi(A)) T[\mu I-\varphi(A)] R(\mu ; \varphi(A)) x \\
& =T R(\mu ; \varphi(A)) x
\end{aligned}
$$

or, $R(\mu ; \varphi(A)) \in R$. Next, using the terminology and notation of $\S 2$, let $m^{\prime} \in W^{\prime}, a\left(m^{\prime}\right)=\lambda$ where $\lambda \in \sigma[A]$. We can certainly find $f \in D\left(\varphi\left(A_{w}\right)\right)$ such that $\varphi(\lambda ; f) \neq 0$. Since

$$
R(\mu ; \varphi(A))\left[\mu \theta(f)-\theta\left(\varphi\left(A_{w}\right) f\right)\right]=\theta(f)
$$

we have

$$
R(\mu ; \phi(A))\left(m^{\prime}\right)\left[\mu \theta(f)-\theta\left(\varphi\left(A_{w}\right) f\right]\left(m^{\prime}\right)=\theta(f)\left(m^{\prime}\right) .\right.
$$

Or,

$$
R(\mu ; \varphi(A))\left(m^{\prime}\right)[\mu-\varphi(\lambda)]=1 .
$$

Hence $\mu \neq \varphi(\lambda)$ for any $\lambda \in \sigma[A]$, which proves (4.3).

To prove (4.4), we note first that if $R\left(\mu ; \varphi\left(A_{w}\right)\right) \in L(w)$ since

$$
\phi\left(\lambda ; R\left(\mu ; \varphi\left(A_{w}\right)\right)=[\mu-\varphi(\lambda)]^{-1}\right.
$$


the complement of the set $\varphi(\sigma[A])$ is not empty. Let $\gamma$ belong to the complement of $\varphi(\sigma[A])$. Now for $m \in W, m \sim \lambda$,

$$
\left[E_{0}+(\gamma-\mu) R\left(\mu ; \varphi\left(A_{w}\right)\right)\right](m)=\frac{\gamma-\varphi(\lambda)}{\mu-\varphi(\lambda)} .
$$

For $m \in U, R\left(\mu ; \varphi\left(A_{w}\right)\right)(m)=0$, so that

$$
\left[E_{0}+(\gamma-\mu) R\left(\mu ; \varphi\left(A_{w}\right)\right)\right](m)=1 .
$$

Hence $\left[E_{0}+(\gamma-\mu) R\left(\mu ; \varphi\left(A_{w}\right)\right)\right]$ has an inverse in $\mathcal{S}(w)$, and it is readily verified that

$$
R\left(\mu ; \varphi\left(A_{w}\right)\right)\left[E_{0}+(\gamma-\mu) R\left(\mu ; \varphi\left(A_{w}\right)\right)\right]^{-1}
$$

is indeed $R\left(\gamma ; \varphi\left(A_{w}\right)\right)$. By Theorem 4.1, $\gamma \in[\varphi(A)]$, proving (4.4).

We may note that (4.4) is not true in general. It is known for instance that (see [1]) we can find $\alpha \in \delta(w), \varphi(\lambda)=\phi(\lambda ; \alpha)$ for which (4.4) does not hold.

It would appear that it is possible to obtain results relating to the fine structure of the spectra of $\varphi(A)$ similar to those in the Hille-Phillips calculus, but we shall not pursue this here.

5. Semigroups in $\delta(w)$. In this section we study semigroups of convolution operators i.e., semigroups of elements in $\delta(w)$ considered as operators over $L(w)$. Let $\alpha(t)$ be a one-parameter semigroup of elements in $\delta(w)$ such that:

(i) $\alpha\left(t_{1}\right) * \alpha\left(t_{2}\right)=\alpha\left(t_{1}+t_{2}\right), t_{1}, t_{2}>0$.

(F) $\quad \alpha(0)=E_{0}$,

(ii) For any $f \in L(w)$,

$$
\|\alpha(t) * f-f\| \rightarrow 0, \text { as } t \rightarrow 0 .
$$

Let $S(t)=\theta(\alpha(t))$. Then as Phillips [3] has shown, $S(t)$ is a strongly continuous semigroup over $X$. The generation of such semigroups is of importance in several contexts, especially in stochastic process theory. The extended operational calculus we have been describing provides a natural setting for the study of these semigroups, and in particular, for determining the relationship of the infinitesimal generator of $S(t)$ to that of $T(\xi)$. We begin with a basic result in this direction.

THEOREM 5.1. Let $\alpha(t)$ be a semigroup in $\mathrm{s}(w)$ satisfying $(\mathrm{F})$. Let $S(t)$ $=\theta(\alpha(t))$ and $\varphi(\lambda)=\log \phi(\lambda ; \alpha(1))$. Then $S(t)$ is a strongly continuous semigroup over $X . \varphi(\lambda) \in \mathfrak{M}$, and the infinitesimal generator of $S(t)$ is $\varphi(A)$.

Proof. $\theta$ being a homomorphism, $S(t)$ is obviously a semigroup. The strong continuity at the origin is immediate from Theorem 3.2. The condition (F) implies that $\varphi(\lambda ; \alpha(t))$ is continuous and multiplicative in $t$ for every $\lambda$, 
$\operatorname{Re} \lambda \leqq w^{+}$. Hence we have:

$$
\phi(\lambda ; \alpha(t))=\operatorname{Exp} t \varphi(\lambda) .
$$

Let $B_{w}$ denote the infinitesimal generator of the semigroup $\alpha(t)$ considered as operators over $L(w)$. Then if $f \in D\left(B_{w}\right)$ it is easy to see that

$$
\phi\left(\lambda ; B_{w} f\right)=\varphi(\lambda) \phi(\lambda ; f) .
$$

Conversely, suppose there is an $f \in L(w)$ and $g \in L(w)$ such that

$$
\phi(\lambda ; g)=\varphi(\lambda) \phi(\lambda ; f) .
$$

Since $B_{w}$ is an infinitesimal generator, $D\left(B_{w}\right)$ is dense. Let $\left\{h_{n}\right\}$ be a sequence in $D\left(B_{w}\right)$ approximating the identity strongly. Now

$$
\phi\left(\lambda ; B_{w}\left(h_{n} * f\right)\right)=\varphi(\lambda) \phi\left(\lambda ; h_{n}\right) \phi(\lambda ; f)=\phi\left(\lambda ; h_{n} * g\right) .
$$

Hence

$$
B_{w}\left(h_{n} * f\right)=h_{n} * g \text {. }
$$

$B_{w}$ being closed, this shows that $f \in D\left(B_{w}\right)$. Also $D\left(B_{w}\right)$ being dense, $\varphi(\lambda) \in \mathscr{M}$, and further, $B_{w}=\varphi\left(A_{w}\right)$. Again, for $f \in D\left(B_{w}\right)$ entirely analogous to (2.4),

$$
\theta\left(B_{w} f\right) x=B \theta(f) x
$$

where we have denoted the infinitesimal generator of $S(t)$ by $B$. Hence

$$
\varphi(A) \theta\left(h_{n}\right) x=\theta\left(B_{w} h_{n}\right) x=B \theta\left(h_{n}\right) x
$$

and for $x \in D(\varphi(A))$ therefore, using (3.7),

$$
\varphi(A) x=\operatorname{limit} \varphi(A) \theta\left(h_{n}\right) x=B x .
$$

For $x \in D(B)$, since $S(t)$ commutes with $\theta\left(h_{n}\right)$ for every $n$,

$$
\theta\left(h_{n}\right) B x=B \theta\left(h_{n}\right) x .
$$

Hence

$$
B x=\operatorname{limit} \theta\left(h_{n}\right) B x=\operatorname{limit} B \theta\left(h_{n}\right) x=\operatorname{limit} \varphi(A) \theta\left(h_{n}\right) x=\varphi(A) x .
$$

Or, $B=\varphi(A)$.

Given a one-parameter family of elements in $\delta(w)$ it is of interest to determine conditions under which it forms a semigroup satisfying $(\mathrm{F})$. We now state a simple sufficient criterion.

TheOREM 5.2. Let $\alpha(t), t>0$, be a one-parameter family of elcments in $\$(w)$ such that:

(i) $\phi\left(\lambda ; \alpha\left(t_{1}+t_{2}\right)\right)=\phi\left(\lambda ; \alpha\left(t_{1}\right)\right) \phi\left(\lambda ; \alpha\left(t_{2}\right)\right), t_{1}, t_{2}>0$.

(ii) $\phi(\lambda ; \alpha(t)) \rightarrow 1$, uniformly in each compact subset of $\operatorname{Re} \lambda \leqq w^{+}$, as $t \rightarrow 0$.

(iii) $\|\alpha(t)\| \rightarrow 1$, as $t \rightarrow 0$.

Then $\alpha(t)$ satisfies $(\mathrm{F})$. 
Proof. Condition (i) suffices trivially to insure the semigroup property. The strong continuity will follow from the following

LEMMA 5.1. Let $\left\{\alpha_{n}\right\}$ be a sequence of elements in $\$(w)$ such that $\phi\left(\lambda ; \alpha_{n}\right) \rightarrow 1$ uniformly in each compact subset of $\operatorname{Re} \lambda \leqq w^{+}$and $\left\|\alpha_{n}\right\| \rightarrow 1$. Then $\alpha_{n}$ approximates the identity strongly over $L(w)$.

Proof. Letting $\left|\alpha_{n}\right|$ denote the variation of $\alpha_{n}$, for any $L>0$, we have

$$
\lim \sup \int_{0}^{L} \exp w(\xi) d_{\xi}\left|\alpha_{n}\right| \leqq \lim \left\|\alpha_{n}\right\|=1 .
$$

Since $\exp w(\xi) \geqq \exp \sigma \xi$ for $-\infty<\sigma \leqq w^{+}$, it follows that

$$
\int_{0}^{L} d_{\xi}\left|\alpha_{n}\right| \leqq M_{0}(L)<\infty .
$$

Hence if $F_{n}(\xi)=\alpha_{n}[0, \xi)$, for $0<\xi \leqq L$,

$$
F_{n}(\xi) \leqq \int_{0}^{L} d_{\xi}\left|\alpha_{n}\right| \leqq M_{0}(L)
$$

or, $F_{n}(\xi)$ is bounded in every closed bounded interval. Again from the wellknown Helly-Bray theorem it follows readily that for every $\xi>0, F_{n}(\xi) \rightarrow 1$. Let $h(\xi)$ be the characteristic function of the interval $[0, h]$. Then $h(\cdot) \in L(w)$ and by a direct computation:

$$
\begin{aligned}
\left\|\alpha_{n} * h-h\right\|= & \int_{0}^{h}\left|F_{n}(\xi)-1\right| \exp w(\xi) d \xi \\
& +\int_{h}^{\infty}\left|F_{n}(\xi)-F_{n}(\xi-h)\right| \exp w(\xi) d \xi .
\end{aligned}
$$

Since $\exp w(\xi)$ is bounded in $[0, h]$, and the $F_{n}(\xi)$ are also bounded therein, the first integral goes to zero. As for the second integral, we note first that

$$
\begin{aligned}
\left\|\alpha_{n} * h\right\| & =\int_{0}^{h}\left|F_{n}(\xi)\right| \exp w(\xi) d \xi+\int_{h}^{\infty}\left|F_{n}(\xi)-F_{n}(\xi-h)\right| \exp w(\xi) d \xi \\
& \leqq\left\|\alpha_{n} \mid\right\| h\|=\| \alpha_{n} \| \int_{0}^{h} \exp w(\xi) d \xi .
\end{aligned}
$$

Hence

$$
\begin{aligned}
\int_{h}^{\infty}\left|F_{n}(\xi)-F_{n}(\xi-h)\right| \exp w(\xi) d \xi & \\
& \leqq\left\|\alpha_{n}\right\| \int_{0}^{h} \exp w(\xi) d \xi-\int_{0}^{h}\left|F_{n}(\xi)\right| \exp w(\xi) d \xi .
\end{aligned}
$$


Since in the larger member of this inequality, $\left\|\alpha_{n}\right\| \rightarrow 1$, and

$$
\int_{0}^{h}\left|F_{n}(\xi)\right| \exp w(\xi) d \xi \rightarrow \int_{0}^{h} \exp w(\xi) d \xi
$$

it follows that the second integral in (5.2) goes to zero also. The linear extension of the class of step functions $h(\cdot)$ being dense in $L(w)$, the BanachSteinhaus theorem applies and shows that the sequence $\left\{\alpha_{n}\right\}$ approximates the identity strongly.

Corollary. Let $\alpha(t)$ be as in the theorem with $S(t)=\theta(\alpha(t))$. Then $\|S(t)\| \rightarrow 1$ also as $t \rightarrow 0$.

Proof. Since $\|S(t)\| \leqq\|\alpha(t)\|$, it follows from (iii) that lim $\sup _{t \rightarrow 0}\|S(t)\| \leqq 1$. On the other hand, the strong continuity implies that $\lim \inf _{t \rightarrow 0}\|S(t)\| \geqq 1$, or $\|S(t)\| \rightarrow 1$.

We may note that condition (iii) is not necessary in order that $\alpha(t)$, be strongly continuous, as may be seen by taking $\alpha(t)=E_{t}$, choosing $\|T(\xi)\|$ discontinuous at the origin.

The more general problem of characterizing multipliers $\varphi(\lambda)$ which are infinitesimal generators of semigroups in $\delta(w)$ satisfying $(F)$ is a natural one to consider in this context. If we restrict ourselves to semigroups of measures, the Kolmogoroff-Levy representation theorem for infinitely divisible distributions (see $[1$, p. 435 , for example]) is readily seen to be a special result of this kind, and has been generalized to semigroups of measures in $\delta(w)$ by Phillips [3].

6. An application. We conclude with an application of the extended calculus to the problem of defining nonintegral powers of the infinitesimal generator of a semigroup. Hille [1, p. 276] has given a method of doing this for a bounded operator with a suitably restricted spectrum. The method to be presented here is different, and it is doubtful that Hille's construction can be carried over to unbounded operators.

For the purposes of this section we shall specialize $T(\xi)$ and suppose in addition to $(\mathrm{H})$ that

$$
\text { (iv) } \int_{1}^{\infty}\|T(\xi)\| \xi^{-\sigma_{0}-1} d \xi<\infty . \quad \text { for some } \sigma_{0}, 0<\sigma_{0}<1
$$

$$
w^{+}=0 \text {. }
$$

Condition $(Q)$ is satisfied for example if we take $\|T(\xi)\|=\log (e+\xi)$ or, $\|T(\xi)\|=(1+\xi)^{\sigma}$ for $0 \leqq \sigma<\sigma_{0}$.

While the definitions below will be given specifically for the semigroup $T(\xi)$, it will be apparent that they will also apply to any strongly continuous semigroup majorized by $T(\xi)$. In particular if $w^{+}<0$, the considerations are simplified greatly. (See [5].) 
Theorem 6.1. For $\lambda$ such that $\operatorname{Re} \lambda \leqq 0$, let

$$
\varphi(\lambda ; \zeta)=\operatorname{Exp}(\zeta \log (-\lambda))
$$

where the principal value of the logarithm is taken (i.e., $\log z$ is real for $z$ positive), and $\zeta=\sigma+i \beta, n \sigma_{0} \leqq \sigma \leqq n$, for positive integral $n$. Then $\varphi(\lambda ; \zeta) \in \mathscr{T}$ (actually $\mathfrak{T}_{\infty}$ ). Further, for $\zeta$ satisfying in addition

$$
\sigma^{2}+\beta^{2}<2 \sigma
$$

we have:

$$
R\left(\mu ; \varphi\left(A_{w} ; \zeta\right)\right) \in L(w),
$$$$
\mu \neq \varphi(\lambda ; \zeta) .
$$

Proof. Let $\sigma_{0} \leqq \sigma<1$. Letting $C_{w} \sim \varphi(\lambda ; \zeta)$, we shall show first that $D\left(C_{w}\right)$ $\supset D\left(A_{w}\right)$. For this we note first that $R\left(1 ; A_{w}\right) \in L(w)$ and that

$$
\phi\left(\lambda ; R\left(1 ; A_{w}\right)\right)=(1-\lambda)^{-1}, \quad \operatorname{Re} \lambda \leqq 0 .
$$

Hence to show that $D\left(C_{w}\right) \supset D\left(A_{w}\right)$ it is obviously enough to show that there is an element $h \in L(w)$ such that

$$
\phi(\lambda ; h)=(1-\lambda)^{-1} \varphi(\lambda ; \zeta) .
$$

Now, by a direct computation (details may be found in [5]) it can be shown that there is a function in $L_{1}[0, \infty)$ whose Laplace transform is given by the right side of (6.3) and further this function is $O\left(\xi^{-\sigma-1}\right)$ as $\xi \rightarrow \infty$, so that it actually belongs to $L(w)$.

For $\zeta=1+i \beta$, a direct evaluation similarly shows that there is an element $g \in L(w)$ such that

$$
\phi(\lambda ; g)=(1-\lambda)^{-2} \varphi(\lambda ; \zeta)
$$

so that $D\left(C_{w}\right) \supset D\left(A_{w}^{2}\right)$, or, $\varphi(\lambda ; \zeta) \in \mathbb{M}_{\infty}$ again. For other values of $\zeta$, since $\zeta$ can by hypothesis always be expressed:

$$
\zeta=n \gamma+i \beta,
$$$$
\sigma_{0} \leqq \gamma \leqq 1
$$

it follows readily from Theorem 3.4 that $\varphi(\lambda ; \zeta) \in \mathfrak{M}_{\infty}$ also.

To prove (6.2) next, it is enough to show that

$$
\phi(\lambda)=[\mu-\varphi(\lambda ; \zeta)]^{-1},
$$

$\operatorname{Re} \lambda \leqq 0$

is the Laplace transform of an element in $L(w)$. A direct estimation again verifies this to be the case. (Details may be found in [5].) This proves the theorem.

We next define:

$$
(-A)^{\zeta}=\varphi(A ; \zeta)
$$

This we may term the "principal value" of $(-A)^{5}$ corresponding to the principal determination of $(-\lambda)^{5}$ taken in (6.1). It is clear that this definition 
interpolates the integral powers of $(-A)$, and so of course will

$$
\varphi(A ; \zeta) \exp 2 \pi i k \zeta
$$

for integral values of $k$. Other properties of these powers follow.

THEOREM 6.2. The operators $(-A)^{5}$ defined by (6.4) [with $\zeta$ values limited as in Theorem 6.1] have the following properties:

$$
\begin{aligned}
(-A)^{\zeta+\gamma} & =(-A)^{\zeta}(-A)^{\gamma}, \\
\sigma\left[(-A)^{\zeta}\right] & \supset[\sigma(-A)]^{\zeta} .
\end{aligned}
$$

For $\sigma^{2}+\beta^{2}<2 \sigma$, and any positive integer $n$,

$$
\sigma\left[(-A)^{n \zeta}\right]=[\sigma(-A)]^{n \zeta} .
$$

Further for $\sigma_{0} \leqq \zeta<1,-(-A)^{\zeta}$ is the infinitesimal generator of a strongly continuous semigroup $S(t ; \zeta), 0<t$, over $X$ such that

$$
S(t ; \zeta)=\theta(f(t ; \zeta))
$$

where the $f(t ; \zeta)$ for each $\zeta$ is a semigroup in $t$ of Lévy stable densities $\in L(w)$. Moreover the semigroup $S(t ; \zeta)$ is such that for $\zeta>\sigma_{0}$

$$
\int_{1}^{\infty}\|S(t ; \zeta)\| t^{-\gamma-1} d t<\infty, \quad \text { for } \gamma \geqq \sigma_{0} / \zeta .
$$

Proof. Properties (6.5) and (6.6) follow directly from Theorem 4.2. For $n=1,(6.7)$ follows from (6.2) and Theorem 4.2. For other values of $n$ it follows thence from the known similar result for the spectrum of integral powers of a closed operator with nonempty resolvent set. Proceeding next to the semigroup generating properties, let

$$
\phi(\lambda ; \zeta)=\operatorname{Exp}-\varphi(\lambda ; \zeta), \quad \sigma_{0} \leqq \zeta<1 .
$$

It is known (see [6] for instance) that for each such $\zeta$,

$$
\phi(\lambda ; \zeta)=\int_{0}^{\infty} e^{\lambda \xi} f_{\zeta}(\xi) d \xi
$$

where $f_{\zeta}(\xi)$ is a Lévy stable density function. Further, an estimation (such as in [5]) readily shows that

$$
f_{\zeta}(\xi)=O\left(\xi^{-\gamma-1}\right) \text { as } \xi \rightarrow \infty
$$

so that $f_{\zeta}(\cdot) \in L(w)$. If we define:

$$
f(t ; \zeta ; \xi)=t^{-1 / 5} f_{\zeta}\left(t^{-1 / \zeta \xi),} \quad 0<t, 0<\xi\right.
$$

it follows readily that $f(t ; \zeta ; \cdot)$ is a semigroup in $t$ of elements in $L(w)$ as well. Let 


$$
S(t ; \zeta)=\theta(f(t ; \zeta ; \cdot))
$$

Then $S(t ; \zeta)$ is also a semigroup for each $\zeta$. Moreover it is strongly continuous at the origin. For

$$
\|S(t ; \zeta) x-x\| \leqq \int_{0}^{\infty}\|T(\xi) x-x\| f(t ; \zeta ; \xi) d \xi .
$$

Since $\|T(\xi) x-x\|$ is bounded continuous in $[0,1]$, the weak convergence property of the densities $f(t ; \zeta ; \cdot)$ shows that the integral

$$
\int_{0}^{1}\|T(\xi) x-x\| f(t ; \zeta ; \xi) d \xi
$$

goes to zero with $t$. Further

$$
\int_{1}^{\infty}\|T(\xi) x-x\| f(t ; \zeta ; \xi) d \xi=O(t) \int_{1}^{\infty}\|T(\xi) x-x\| \xi^{-\zeta-1} d \xi
$$

where we have used (6.9). Hence $S(t ; \zeta)$ is strongly continuous at the origiil. That the infinitesimal generator of the semigroup $S(t ; \zeta)$ is $-(-A)^{\zeta}$ fcllows from Theorem 5.1. Finally, a direct computation yields (6.8). Thus

$$
\begin{aligned}
\int_{1}^{\infty} S(t ; \zeta) t^{-\gamma-1} d t & =\int_{1}^{\infty} \int_{0}^{\infty}\left\|T\left(\xi t^{1 / \zeta}\right)\right\| f_{\zeta}(\xi) t^{-\gamma-1} d t d \xi \\
& =O(1)+\int_{1}^{\infty} \int_{1}^{\infty}\left\|T\left(\xi t^{1 / \zeta}\right)\right\| f_{\zeta}(\xi) t d t d \xi \\
& =O(1)+\int_{1}^{\infty} f_{\zeta}(\zeta) \xi^{\gamma \zeta} d \xi \int_{\xi}^{\infty}\|T(t)\| t^{-\gamma \zeta-1} d t \\
& \leqq O(1)+\int_{1}^{\infty} f_{\zeta}(\xi) \xi^{\sigma_{0}} d \xi \int_{\xi}^{\infty}\|T(t)\| t^{-\sigma_{0}-1} d t \\
& <\infty .
\end{aligned}
$$

The connection between the operators $(-A)^{5}$ as defined by (6.4) using the extended calculus, and the Riemann-Liouville fractional integrals (which may be expected owing to the similarity between infinitesimal generators and differential operators) is made precise in the following theorem.

Theorem 6.3. The operators $(-A)^{5}$ defined by (6.4) have the following alternate representation:

$$
\begin{aligned}
(-A)^{\zeta} x & =\frac{1}{\Gamma(-\zeta)} \int_{0}^{\infty}\left[T(\xi) x-\sum_{0}^{n-1} \frac{A^{k} x \xi^{k}}{k !}\right] \xi^{-\zeta-1} d \xi \\
\text { for } x & \in D\left(A^{n}\right), \quad n-1+\sigma_{0} \leqq \operatorname{Re} \zeta<n
\end{aligned}
$$


where the integral on the right is a Bochner integral.

Proof. Let $S(\xi)$ be any (strongly continuous) semigroup majorized by $T(\xi)$, with infinitesimal generator $B$. For $x \in D\left(B^{n}\right)$, we have $[1$, p. 236]:

$$
S(t) x=\sum_{0}^{n-1} \frac{t^{k} B^{k} x}{k !}+\frac{1}{(n-1) !} \int_{0}^{t}(t-\xi)^{n-1} S(\xi) B^{n} x d \xi
$$

so that the integral

$$
\int_{0}^{\infty}\left[S(t) x-\sum_{0}^{n-1} \frac{t^{k} B^{k} x}{k !}\right] t^{-\zeta-1} d t
$$

exists in the Bochner sense. Specializing to $S(\xi) \equiv E_{\xi}, X=L(w)$ and $f \in D\left(A_{w}^{n}\right)$, let

$$
g=\frac{1}{\Gamma(-\zeta)} \int_{0}^{\infty}\left[E_{t} * f-\sum_{0}^{n-1} \frac{t^{k} A_{w}^{k} f}{k !}\right] t^{-\zeta-1} d t .
$$

Since $\theta$ is a homomorphism,

$$
\theta(g)=\frac{1}{\Gamma(-\zeta)} \int_{0}^{\infty}\left[T(t) \theta(f)-\sum_{0}^{n-1} \frac{t^{k} A^{k} \theta(f)}{k !}\right] t^{-\zeta-1} d t .
$$

Again,

$$
\phi(\lambda ; g)=\frac{1}{\Gamma(-\zeta)} \int_{0}^{\infty}\left[e^{\lambda t}-\sum_{0}^{n-1} \frac{t^{k} \lambda^{k}}{k !}\right] t^{-\zeta-1} d t \phi(\lambda ; f)
$$

which, by a straightforward evaluation of the integral,

$$
=\varphi(\lambda ; \zeta) \phi(\lambda ; f) \text {. }
$$

Hence for $x \in X$,

$$
\theta(g) x=(-A)^{5} \theta(f) x
$$

Further for $x \in D\left(A^{n}\right)$,

$$
\theta(g) x=(-A)^{5} \theta(f) x .
$$

On the other hand using (6.11), for $x \in D\left(A^{n}\right)$,

$$
\theta(g) x=\frac{\theta(f)}{\Gamma(-\zeta)} \int_{0}^{\infty}\left[T(t) x-\sum_{0}^{n-1} \frac{t^{k} A^{k} x}{k !}\right] t^{-\zeta-1} d t .
$$

Hence

$$
(-A)^{\zeta} \theta(f) x=\theta(f) \frac{1}{\Gamma(-\zeta)} \int_{0}^{\infty}\left[T(t) x-\sum_{0}^{n-1} \frac{t^{k} A^{k} x}{k !}\right] t^{-\zeta-1} d t
$$


The domain of $A_{w}^{n}$ being dense, we can use a sequence in it approximating the identity strongly in place of $f$, and since $(-A)^{\zeta}$ is closed, (6.10) follows.

One might inquire at this point whether other determinations of nonintegral powers of $(-A)$ are possible which are not of the form $(-A)^{\zeta} \exp 2 \pi i k \zeta$, $(-A)^{5}$ being the principal value defined by (6.4). The answer to this is in the affirmative (settling a question raised by Hille $[1$, p. 276] in a similar context). For this, one has only to take $T(\xi)$ to be a continuous group such that $\|T(\xi)\| \leqq M$. Specifically, we may take the group of translations over $L_{1}(-\infty, \infty)$. Then since $(-A)$ is the infinitesimal generator of $T(-\xi)$, we can define $(A)^{5}$ as well as $(-A)^{\zeta}$ using (6.4), and it is easily seen that

$$
(A)^{\zeta} \exp \pi i \zeta \neq(-A)^{\zeta} \exp 2 \pi i k \zeta
$$

for any integer $k$, although $(A)^{5} \exp \pi i \zeta$ certainly interpolates integral powers of $(-A)$.

On the other hand, the principal value as defined by (6.4) has an extremal property similar to the one given by Hille [1, p. 276]. To avoid lengthy details, we may assume now that the semigroup $T(\xi)$ is such that it satisfies a stronger version of $(Q)$, viz:

$$
\int_{1}^{\infty}\|T(\xi)\| \xi^{-\sigma-1} d \xi<\infty, \text { for every } \sigma, \quad 0<\sigma<1 .
$$

Then obviously, $(-A)^{\zeta}$ may be defined by (6.4) for every $\zeta$ such that $\operatorname{Re} \zeta>0$, and will further have the representation (6.10) where now the $\sigma_{0}$ is taken to be zero. Moreover, for any $x \in D\left(A^{\infty}\right)$ it can be shown that $(-A)^{5} x$ is analytic in $\zeta$ for $\operatorname{Re} \zeta>0$. (Details may be found in [5].) Moreover, a simple computation using (6.10) shows that

$$
\limsup _{|\eta| \rightarrow \infty} \frac{\log \left\|(-A)^{\sigma+i \eta} x\right\|}{|\eta|} \leqq \pi / 2, \quad 0<\sigma .
$$

We now assert that it is not possible to find a determination preserving analyticity for $x \in D\left(A^{\infty}\right)$ and interpolating integral powers, and having the property (6.14), other than that given by (6.4). For, if there were one, an application of a classical result of F. Carlson (as in Hille [1, p. 277]) shows that it must coincide with (6.4).

Acknowledgment. It is a pleasure to acknowledge the numerous suggestions and criticism of Professor R. S. Phillips in the preparation of this paper. The author was also privileged to read in manuscript parts of the American Mathematical Society Colloquium publication on semigroup theory by $\mathrm{E}$. Hille and R. S. Phillips.

\section{REFERENCES}

1. E. Hille, Functional analysis and semigroups, Amer. Math. Soc. Colloquium Publications, vol. $31,1948$. 
2. R. S. Phillips, Semi-groups of operators, Bull. Amer. Math. Soc. vol. 61 (1955) pp. 16-33

3. - On the generation of semigroups of linear operators, Pacific J. Math. (1952) pp 343-369.

4. - Spectral theory for semigroups of linear operators, Trans. Amer. Math. Soc. vol. 71 (1951) pp. 393-415.

5. A. V. Balakrishnan, On powers of the infinitesimal generators of groups and semigroups of linear bounded transformations, Thesis, University of Southern California, 1954.

6. B. V. Gnedenko and A. N. Kolmogoroff, Limit distributions of sums of independent random variables, Addison Wesley Publishing Company, 1954.

University of Southern California,

Los Angeles, Calif. 